\title{
The bounded approximation property for the predual of the space of bounded holomorphic mappings
}

\author{
by \\ ERHAN ÇALIŞKAN (Istanbul)
}

\begin{abstract}
When $U$ is the open unit ball of a separable Banach space $E$, we show that $G^{\infty}(U)$, the predual of the space of bounded holomorphic mappings on $U$, has the bounded approximation property if and only if $E$ has the bounded approximation property.
\end{abstract}

1. Introduction. Let $E$ and $F$ be complex Banach spaces, and let $L(E ; F)$ be the Banach space of all continuous linear operators. Let $1 \leq$ $\lambda<\infty$. A Banach space $E$ is said to have:

- the approximation property (AP for short) if given a compact set $K \subset$ $E$ and $\varepsilon>0$, there is a finite rank operator $T \in L(E ; E)$ such that $\|T x-x\|<\varepsilon$ for every $x \in K$;

- the $\lambda$-bounded approximation property ( $\lambda$-BAP for short) if given a compact set $K \subset E$ and $\varepsilon>0$, there is a finite rank operator $T \in$ $L(E ; E)$ so that $\|T\| \leq \lambda$ and $\|T x-x\|<\varepsilon$ for every $x \in K$;

- the bounded approximation property (BAP for short) if it has the $\lambda$ BAP for some $\lambda$;

- the metric approximation property (MAP for short) if it has the 1-BAP.

Clearly the BAP implies the AP. But the converse is in general false. In [5] Figiel and Johnson gave an example of a separable Banach space with the AP which fails to have the BAP. (See also Casazza [2].)

Let $U$ be an open subset of a Banach space $E$, and let $G^{\infty}(U)$ denote the predual of the space $\mathcal{H}^{\infty}(U)$ of all bounded holomorphic mappings constructed by Mujica in [10]. If $U$ is a bounded balanced open subset of $E$ then Mujica [10] proved that $E$ has the AP (resp. MAP) if and only if $G^{\infty}(U)$ has the AP (resp. MAP). In [1] the author proved that $E$ has the compact AP if and only if $G^{\infty}(U)$ has the compact AP.

2000 Mathematics Subject Classification: 46G20, 46B28, 46G25, 46E50.

Key words and phrases: Banach spaces, bounded approximation property, bounded holomorphic mappings. 
In the present paper, we establish our notation and terminology in Section 2, and in Section 3 we give two basic results, one of which characterizes the weakly compactly generated Banach spaces with the BAP. In Section 4 we show that a Banach space $E$ has the $\lambda$-BAP if and only if each $m$ homogeneous continuous Banach-valued polynomial on $E$ of norm $\leq 1$ can be uniformly approximated on compact sets by $m$-homogeneous finite rank polynomials of norm $\leq \lambda^{m}$, for some $1 \leq \lambda<\infty$. In our main result we show that a separable Banach space $E$ has the BAP if and only if $G^{\infty}(U)$ has the BAP, where $U$ is the open unit ball of $E$. This gives a partial solution for a problem posed by Mujica in [10].

I wish to thank Professor Jorge Mujica for many useful suggestions on the exposition.

2. Notation and terminology. The symbol $\mathbb{C}$ represents the field of all complex numbers, $\mathbb{N}$ the set of all positive integers, and $\mathbb{N}_{0}=\mathbb{N} \cup\{0\}$. Unless stated otherwise $E$ and $F$ denote complex Banach spaces. The letter $U$ denotes a nonvoid open subset of $E$, and $U_{E}$ is the open unit ball of $E$.

The symbol $B_{E}^{\lambda}, 1 \leq \lambda<\infty$, represents the subset of $E$ consisting of the elements of norm $\leq \lambda$. We write $B_{E}$ instead of $B_{E}^{1}$, the closed unit ball of $E$.

The symbol $\tau_{\mathrm{c}}$ denotes the compact-open topology.

Let $L(E ; F)$ denote the Banach space of all continuous linear operators from $E$ into $F$. When $F=\mathbb{C}$ we write $E^{\prime}$ instead of $L(E ; \mathbb{C})$.

We denote by $E \otimes F$ the tensor product of $E$ and $F$.

Let $\mathcal{P}(E ; F)$ denote the vector space of all continuous polynomials from $E$ into $F$, and $\mathcal{P}\left({ }^{m} E ; F\right)$ the subspace of all $m$-homogeneous members of $\mathcal{P}(E ; F)$. If $F=\mathbb{C}$ then we denote $\mathcal{P}\left({ }^{m} E ; \mathbb{C}\right)$ by $\mathcal{P}\left({ }^{m} E\right)$.

A polynomial $P \in \mathcal{P}\left({ }^{m} E ; F\right)$ is said to be of finite type if it is a linear combination of functions $\phi^{m} \otimes y\left(\phi \in E^{\prime}, y \in F\right)$, where $\phi^{m} \otimes y(x)=\phi^{m}(x) y$ for all $x \in E$. Let $\mathcal{P}_{\mathrm{f}}\left({ }^{m} E ; F\right)$ denote the subspace of all members of $\mathcal{P}\left({ }^{m} E ; F\right)$ which are of finite type, for every $m \in \mathbb{N}_{0}$.

We denote by $\mathcal{H}^{\infty}(U ; F)$ the Banach space of all bounded holomorphic mappings from $U$ into $F$, with the supremum norm. When $F=\mathbb{C}$ we write $\mathcal{H}^{\infty}(U)$ instead of $\mathcal{H}^{\infty}(U ; \mathbb{C})$.

We refer to [3] or [9] for the properties of polynomials and holomorphic mappings on infinite-dimensional spaces, and to [7] for the theory of Banach spaces.

3. The bounded approximation property in Banach spaces. An operator $T$ in $L(E ; F)$ is said to have a finite rank if $T(E)$ is finite-dimensional. Observe that the subspace of all finite rank operators $T \in L(E ; F)$ can be identified with the space $E^{\prime} \otimes F$. 
In [7, Theorem 1.e.4] Lindenstrauss and Tzafriri give a list of equivalent formulations of the AP for Banach spaces. The following analogous proposition characterizing spaces with the BAP can be proved easily (see also [2]).

Proposition 1. Let $E$ be a Banach space and $1 \leq \lambda<\infty$. The following statements are equivalent:

(a) $E$ has the $\lambda$-BAP.

(b) $B_{L(E ; E)}=\overline{B_{E^{\prime} \otimes E}^{\lambda}} \tau_{\mathrm{c}}$.

(c) For every Banach space $F, B_{L(F ; E)}={\overline{B_{F^{\prime} \otimes E}^{\lambda}}}_{\tau_{c}}$.

(d) For every Banach space $F, B_{L(E ; F)}=\overline{B_{E^{\prime} \otimes F}^{\lambda}} \tau_{c}$.

(e) For every $c>0$, every $\left(x_{n}\right)_{n=1}^{\infty} \subset E$, and $\left(x_{n}^{\prime}\right)_{n=1}^{\infty} \subset E^{\prime}$ with $\sum_{n=1}^{\infty}\left\|x_{n}\right\| \cdot\left\|x_{n}^{\prime}\right\|<\infty \quad$ and $\quad\left|\sum_{n=1}^{\infty} x_{n}^{\prime}\left(T x_{n}\right)\right| \leq c \quad$ for all $T \in B_{E^{\prime} \otimes E}^{\lambda}$, we have $\left|\sum_{n=1}^{\infty} x_{n}^{\prime}\left(x_{n}\right)\right| \leq c$.

A common generalization of the notions of a reflexive and a separable Banach space is given in the following definition.

DeFinition 1. A Banach space $E$ is said to be weakly compactly generated (WCG for short) if there is a weakly compact subset $K$ of $E$ such that $E=\overline{\bigcup_{n=1}^{\infty} n K}$.

For examples of WCG Banach spaces other than separable and reflexive spaces see [6].

One can easily show that if $E$ has the $\lambda$-BAP, $1 \leq \lambda<\infty$, then every complemented subspace of $E$ with the projection $P$ has the $\lambda\|P\|$-BAP. Recall that a complemented subspace of $E$ is said to be 1-complemented if the corresponding projection has norm 1. Using Proposition 1 and [6, Theorem 2.1] we easily get the following characterization of the BAP for WCG Banach spaces, which we have not found in the literature.

Corollary 1. Let $E$ be a WCG Banach space, and $1 \leq \lambda<\infty$. The following are equivalent:

(a) E has the $\lambda$-BAP.

(b) Every 1-complemented subspace of $E$ has the $\lambda$-BAP.

(c) Every 1-complemented separable subspace of $E$ has the $\lambda$-BAP.

One can also obtain results similar to Proposition 1 and Corollary 1 for the bounded compact approximation property (for the definition see [2]).

4. The bounded approximation property for the predual of the space of bounded holomorphic mappings. In [13] Ryan constructed a Banach space $Q\left({ }^{m} E\right), m \in \mathbb{N}$, and a mapping $\delta_{m} \in \mathcal{P}\left({ }^{m} E ; Q\left({ }^{m} E\right)\right)$ 
with the following universal property: For each Banach space $F$ and each $P \in \mathcal{P}\left({ }^{m} E ; F\right)$, there is a unique operator $T_{P} \in L\left(Q\left({ }^{m} E\right) ; F\right)$ such that $T_{P} \circ \delta_{m}=P$. The space $Q\left({ }^{m} E\right)$ is defined as the closed subspace of all linear functionals $v \in \mathcal{P}\left({ }^{m} E\right)^{\prime}$ such that $\left.v\right|_{B_{\mathcal{P}\left(m_{E}\right)}}$ is $\tau_{\mathrm{c}}$-continuous; it is called the predual of $\mathcal{P}\left({ }^{m} E\right)$. (See also [11], or [10, Theorems 2.4 and 4.1].)

The next result asserts that a Banach space $E$ has the BAP if and only if, for each $m \in \mathbb{N}, Q\left({ }^{m} E\right)$ has the BAP.

Proposition 2. Let $E$ be a Banach space and let $1 \leq \lambda<\infty$. The following statements are equivalent:

(a) E has the $\lambda$-BAP.

(b) For each Banach space $F$ and each $m \in \mathbb{N}, B_{\mathcal{P}\left({ }^{m} E ; F\right)}={\overline{B_{\mathcal{P}_{f}\left({ }^{m} E ; F\right)}}}_{\tau_{\mathrm{c}}}$.

(c) For each $m \in \mathbb{N}, Q\left({ }^{m} E\right)$ has the $\lambda^{m}-B A P$.

Proof. The proof of $(\mathrm{a}) \Rightarrow(\mathrm{b})$ is standard while $(\mathrm{b}) \Rightarrow(\mathrm{c})$ is a consequence of [10, Theorem 2.4 and Proposition 3.1]. Finally, (c) $\Rightarrow$ (a) follows from the fact that $Q\left({ }^{1} E\right)=E($ see $[10$, p. 871]).

We note that the implication $(\mathrm{a}) \Rightarrow(\mathrm{c})$ was stated in $[10, \mathrm{p} .885]$ without proof.

The space $\mathcal{H}^{\infty}(U)$ also has a predual. In [10] Mujica constructed a Banach space $G^{\infty}(U)$ and a mapping $\delta_{U} \in \mathcal{H}^{\infty}\left(U ; G^{\infty}(U)\right)$ with the following universal property: For each Banach space $F$ and each mapping $f \in \mathcal{H}^{\infty}(U ; F)$, there is a unique operator $T_{f} \in L\left(G^{\infty}(U) ; F\right)$ such that $T_{f} \circ \delta_{U}=f$. The space $G^{\infty}(U)$ is defined as the closed subspace of all linear functionals $u \in \mathcal{H}^{\infty}(U)^{\prime}$ such that $\left.u\right|_{B_{\mathcal{H}^{\infty}(U)}}$ is $\tau_{\mathrm{c}}$-continuous; it is called the predual of $\mathcal{H}^{\infty}(U)$.

Now it is natural to ask whether there is a result similar to Proposition 2 for $G^{\infty}(U)$ concerning the BAP. In fact, in [10] Mujica posed the following problem (see [10, 5.9 Problem]): Does $G^{\infty}\left(U_{E}\right)$ have the BAP whenever $E$ does? We have no answer to this question in general, but we give a positive answer if $E$ is separable. First we give a general relation between $E$ and $G^{\infty}(U)$ in connection with the BAP.

If $U$ is a bounded open subset of a Banach space $E$, then by [10, Proposition 2.3], $E$ is topologically isomorphic to a complemented subspace of $G^{\infty}(U)$. In the following proposition $P \in L\left(G^{\infty}(U) ; E\right)$ will indicate the relevant projection.

Proposition 3. Let $U$ be an open subset of a Banach space $E$ and let $1 \leq \lambda<\infty$. Consider the following statements:

(a) For each Banach space $F, B_{\mathcal{H}^{\infty}(U ; F)}=\overline{B_{\mathcal{H}^{\infty}(U) \otimes F}^{\lambda}} \tau_{\mathrm{c}}$.

(b) $G^{\infty}(U)$ has the $\lambda$-BAP.

(c) E has the $\lambda\|P\|-B A P$. 
(d) For each Banach space $F$ and for each open subset $V \subset F$,

$$
B_{\mathcal{H}^{\infty}(V ; E)}=\overline{B_{\mathcal{H}^{\infty}(V) \otimes E}^{\lambda\|P\|}} \tau_{\mathrm{c}} .
$$

(e) $I_{U} \in{\overline{B_{\mathcal{H}^{\infty}(U) \otimes E}^{\lambda\|P\|}}}_{\tau_{\mathrm{c}}}$.

Then we always have the implications $(\mathrm{a}) \Leftrightarrow(\mathrm{b})$ and $(\mathrm{c}) \Rightarrow(\mathrm{d})$. If , in addition, $U$ is bounded then $(\mathrm{b}) \Rightarrow(\mathrm{c})$ and $(\mathrm{d}) \Rightarrow(\mathrm{c})$. If $U$ is the open unit ball then also $(\mathrm{d}) \Rightarrow(\mathrm{e})$.

Proof. The equivalence (a) $\Leftrightarrow(\mathrm{b})$ follows easily from Proposition 1 and [10, Theorem 2.1, Proposition 3.1 and Proposition 4.9], and (c) $\Rightarrow(d)$ follows from Proposition 1 and [10, Theorem 2.1, Corollary 4.10 and Proposition 3.1].

If $U$ is bounded, we get (b) $\Rightarrow$ (c) from [10, Proposition 2.3]. Now by modifying an idea of Mujica [10, p. 883] we shall show that (d) implies (c). From (d), by using, [10, Theorem 2.1, Proposition 3.1 and Corollary 4.10], we deduce that

$$
B_{L\left(G^{\infty}(U) ; E\right)}=\overline{B_{G^{\infty}(U)^{\prime} \otimes E}^{\lambda\|P\|}} \tau_{\mathrm{c}}
$$

for every Banach space $F$. Let $A \in B_{L(F ; E)}$ be given. By [10, Proposition 2.3] there are operators $S \in B_{L\left(F ; G^{\infty}\left(U_{F}\right)\right)}$ and $S \in B_{L\left(G^{\infty}\left(U_{F}\right) ; F\right)}$ such that $T \circ S(y)=y$ for every $y \in F$. Then $A \circ T \in B_{L\left(G^{\infty}\left(U_{F}\right) ; E\right)}$ and therefore there is a net $\left(B_{\alpha}\right)_{\alpha \in \Lambda} \subset B_{G^{\infty}\left(U_{F}\right)^{\prime} \otimes E}^{\lambda\|P\|}$ which converges to $A \circ T$ for $\tau_{\mathrm{c}}$. Hence $\left(B_{\alpha} \circ S\right)_{\alpha \in \Lambda} \subset B_{F^{\prime} \otimes E}^{\lambda\|P\|}$ and $B_{\alpha} S \rightarrow A \circ T \circ S=A$ for the topology $\tau_{\mathrm{c}}$. Thus, by Proposition 1, $E$ has the $\lambda\|P\|$-BAP.

Finally, if $U$ is the open unit ball of $E$ then (d) $\Rightarrow(\mathrm{e})$ follows from the fact that $I_{U} \in B_{\mathcal{H}^{\infty}(U ; E)}$.

Note that, if $U$ is a bounded open subset of a separable Banach space $E$ which has the AP, but does not have the BAP (see [5]), then $G^{\infty}(U)$ has the AP [10, Theorem 5.4], but does not have the BAP by Proposition 3. We do not know, in the preceding proposition, if the implication $(\mathrm{c}) \Rightarrow(\mathrm{b})$ is true for any Banach space $E$, i.e., whether or not $G^{\infty}(U)$ has the BAP whenever $E$ does. As stated above, we have an affirmative answer for $E$ separable. Before proving this result we need some preparation.

If $E$ has a Schauder basis $\left(e_{n}\right)_{n=1}^{\infty}$ then $E_{n}:=\left\langle e_{1}, \ldots, e_{n}\right\rangle$ denotes the subspace generated by $e_{1}, \ldots, e_{n}$, and $T_{n}: E \rightarrow E_{n}$ the canonical projection, for each $n \in \mathbb{N}$. If $U$ is an open subset of $E$ then we set $U_{n}:=U \cap E_{n}$ for each $n \in \mathbb{N}$.

The following lemma, due to Mujica [8], plays an important role in the proof of Theorem 1 (see also [4, Lemma 3.1]).

Lemma 1 ([8], [4]). Let $E$ be a Banach space with a Schauder basis. Let $U$ be a balanced convex open subset of $E$. Then there are three increas- 
ing sequences $\left(A_{n}\right)_{n=1}^{\infty},\left(B_{n}\right)_{n=1}^{\infty}$ and $\left(C_{n}\right)_{n=1}^{\infty}$ of open subsets of $U$ with the following properties:

(a) $C_{n} \subset B_{n} \subset A_{n}$ for every $n$, and $\bigcup_{n=1}^{\infty} C_{n}=U$.

(b) $T_{n}\left(A_{n}\right) \subset U_{n} \subset A_{n}$ for every $n$.

(c) $B_{j} \cap U_{n}$ is a compact subset of $A_{j} \cap U_{n}$ for every $j$ and $n$.

(d) $T_{n}\left(C_{j}\right) \subset B_{j} \cap U_{n}$ whenever $n \geq j$.

(e) For each compact subset $K$ of $U$ and balanced convex open neighborhood of zero $V$ in $E$ such that $K+V \subset U$, there exists $n_{0} \in \mathbb{N}$ such that $K \subset C_{n}$ and $T_{n}(x) \in x+V$ whenever $x \in K$ and $n \geq n_{0}$.

The proof of the following result was suggested to us by Professor Jorge Mujica. This greatly simplified our original proof.

THEOREM 1. Let E be a Banach space with a monotone Schauder basis. Then $\mathcal{H}^{\infty}\left(U_{E}\right) \otimes F$ is sequentially dense in $\left(\mathcal{H}^{\infty}\left(U_{E} ; F\right), \tau_{\mathrm{c}}\right)$ for every Banach space $F$.

Proof. Let $f \in \mathcal{H}^{\infty}\left(U_{E} ; F\right)$ be given. We may assume, without loss of generality, that $\|f\| \leq 1$. Since, by hypothesis, $E$ has the MAP (see, for example, [7, p. 38]), by [10, Proposition 5.7] we have $B_{\mathcal{H}^{\infty}\left(U_{E} ; F\right)}={\overline{B_{\mathcal{H}^{\infty}\left(U_{E}\right) \otimes F}}}^{\tau_{\mathrm{c}}}$. Hence, there exists a sequence $\left(h_{n}\right)_{n=1}^{\infty} \subset B_{\mathcal{H}^{\infty}\left(U_{E}\right) \otimes F}$ such that

$$
\left\|h_{n}(y)-f(y)\right\|<1 / n \quad \text { for all } y \in B_{n} \cap U_{n} .
$$

Since $\left\|h_{n}\right\| \leq 1$ for every $n$, the sequence $\left(h_{n}\right)_{n=1}^{\infty}$ is equicontinuous (see [9, Proposition 9.15]). It follows from $(*)$ that $\left(h_{n}(y)\right)_{n=1}^{\infty}$ converges to $f(y)$ for every $y \in D=\bigcup_{n=1}^{\infty}\left(B_{n} \cap U_{n}\right)$. As $E$ has the MAP, it is easy to see that $D$ is dense in $U_{E}$ by Lemma 1 . As $\left(h_{n}\right)_{n=1}^{\infty}$ is equicontinuous, it follows that $\left(h_{n}(y)\right)_{n=1}^{\infty}$ is a Cauchy sequence in $F$ for every $y \in U_{E}$ (see the proof of $[14$, Theorem 3]).

Define $h(y)=\lim h_{n}(y)$ for every $y \in U_{E}$. Since $\left(h_{n}\right)_{n=1}^{\infty}$ is equicontinuous, $h$ is continuous (see [9, Lemma 9.10]). Since $h(y)=f(y)$ for every $y \in D$, and $D$ is dense in $U_{E}$, it follows that $h(y)=f(y)$ for every $y \in U_{E}$. Now, from equicontinuity of $\left(h_{n}\right)_{n=1}^{\infty}$, it follows that $\left(h_{n}\right)_{n=1}^{\infty}$ converges to $f$ uniformly on compact subsets of $U_{E}$ (see [9, Proposition 9.11]). This completes the proof.

Using a result of Pełczyński we obtain the following stronger version of the previous theorem.

Corollary 2. Let $E$ be a separable Banach space with the BAP. Then $\mathcal{H}^{\infty}\left(U_{E}\right) \otimes F$ is sequentially dense in $\left(\mathcal{H}^{\infty}\left(U_{E} ; F\right), \tau_{\mathrm{c}}\right)$ for every Banach space $F$.

Proof. By a result of Pełczyński [12] there exists a Banach space $M$ with a Schauder basis, and a Banach space $N$, such that $M=E \oplus N$. We may assume that $M$ has a monotone Schauder basis. Now, $U_{E} \oplus N$ is an open 
subset of $E \oplus N=M$. Let $\sigma: x \in U_{E} \mapsto x+0 \in U_{E} \oplus N$ and let $\pi$ : $x+y \in U_{E} \oplus N \mapsto x \in U_{E}$. If $f \in \mathcal{H}^{\infty}\left(U_{E} ; F\right)$, then $f \circ \pi \in \mathcal{H}^{\infty}\left(U_{E} \oplus N ; F\right)$. By Theorem 1 there exists a sequence $\left(h_{n}\right)_{n=1}^{\infty} \subset \mathcal{H}^{\infty}\left(U_{E} \oplus N\right) \oplus F$ which converges to $f \circ \pi$ in $\left(\mathcal{H}^{\infty}\left(U_{E} \oplus N ; F\right), \tau_{\mathrm{c}}\right)$. Hence the sequence $\left(h_{n} \circ \sigma\right)_{n=1}^{\infty}$ lies in $\mathcal{H}^{\infty}\left(U_{E}\right) \oplus F$, and converges to $f \circ \pi \circ \sigma=f$ in $\left(\mathcal{H}^{\infty}\left(U_{E} ; F\right), \tau_{\mathrm{c}}\right)$.

To attain our main result we need the following proposition which shows that sequential convergence on $\mathcal{H}^{\infty}(U ; F)$ for the topology $\tau_{\mathrm{c}}$ implies sequential convergence on $L\left(G^{\infty}(U) ; F\right)$ for the topology of pointwise convergence.

Proposition 4. Let $U$ be an open subset of a Banach space $E$ and let $F$ be a Banach space. If $\mathcal{H}^{\infty}(U) \otimes F$ is sequentially dense in $\left(\mathcal{H}^{\infty}(U ; F), \tau_{\mathrm{c}}\right)$, then for each $T \in L\left(G^{\infty}(U) ; F\right)$ there exists a sequence $\left(T_{n}\right)_{n=1}^{\infty} \subset G^{\infty}(U)^{\prime}$ $\otimes F$ such that $T_{n}(u) \rightarrow T(u)$ for every $u \in G^{\infty}(U)$.

Proof. Let $T \in L\left(G^{\infty}(U) ; F\right)$ be given. Then by [10, Theorem 2.1] there exists a corresponding mapping $f \in \mathcal{H}^{\infty}(U ; F)$ and hence, by hypothesis, there is a sequence $\left(f_{n}\right)_{n=1}^{\infty} \subset \mathcal{H}^{\infty}(U) \otimes F$ such that $f_{n} \rightarrow f$ for the compact-open topology $\tau_{\mathrm{c}}$. By [10, Theorem 2.1 and Proposition 3.1] there is a corresponding sequence $\left(T_{f_{n}}\right)_{n=1}^{\infty} \subset G^{\infty}(U)^{\prime} \otimes F$.

We claim that $T_{f_{n}}(u) \rightarrow T(u)$ for every $u \in G^{\infty}(U)$. Let $u \in G^{\infty}(U)$ and $\varepsilon>0$ be given. By [10, Corollary 4.12] there exist $\left(\alpha_{i}\right)_{i=1}^{\infty} \subset l_{1}$ and $\left(x_{i}\right)_{i=1}^{\infty} \subset U$ such that $u=\sum_{i=1}^{\infty} \alpha_{i} \delta_{U}\left(x_{i}\right)$, where $\delta_{U}$ is the bounded holomorphic mapping given in [10, Theorem 2.1] for which $T_{f_{n}} \circ \delta_{U}=f_{n}$ for every $n \in \mathbb{N}$, and $T \circ \delta_{U}=f$. Let $m$ be any positive integer and consider $u_{m}:=\sum_{i=1}^{m} \alpha_{i} \delta_{U}\left(x_{i}\right)$. Since $f_{n}$ converges to $f$ for the topology $\tau_{\mathrm{c}}$, and $\left(x_{i}\right)_{i=1}^{m}$ is a compact subset of $U$, there is an $n_{0} \in \mathbb{N}$ such that

$$
\sup _{1 \leq i \leq m}\left\|f_{n}\left(x_{i}\right)-f\left(x_{i}\right)\right\| \leq \frac{\varepsilon}{m \sup _{1 \leq i \leq m}\left|\alpha_{i}\right|}
$$

for all $n \geq n_{0}$. Hence, for each $m \in \mathbb{N}$ we get

$$
\begin{aligned}
\left\|T_{f_{n}}\left(u_{m}\right)-T\left(u_{m}\right)\right\| & =\left\|\sum_{i=1}^{m} \alpha_{i}\left(T_{f_{n}}\left(\delta_{U}\left(x_{i}\right)\right)-T\left(\delta_{U}\left(x_{i}\right)\right)\right)\right\| \\
& \leq \sum_{i=1}^{m}\left|\alpha_{i}\right| \|\left(T_{f_{n}} \circ \delta_{U}\left(x_{i}\right)-T \circ \delta_{U}\left(x_{i}\right) \|\right. \\
& \leq \sup _{1 \leq i \leq m}\left|\alpha_{i}\right| \sum_{i=1}^{m}\left\|f_{n}\left(x_{i}\right)-f\left(x_{i}\right)\right\| \\
& \leq m \sup _{1 \leq i \leq m}\left|\alpha_{i}\right| \frac{\varepsilon}{m \sup _{1 \leq i \leq m}\left|\alpha_{i}\right|}=\varepsilon
\end{aligned}
$$

for all $n \geq n_{0}$. Therefore

$$
\left\|T_{f_{n}}(u)-T(u)\right\|=\lim _{m \rightarrow \infty}\left\|T_{f_{n}}\left(u_{m}\right)-T\left(u_{m}\right)\right\| \leq \varepsilon
$$

for all $n \geq n_{0}$, which completes the proof. 
Now from Corollary 2 and Proposition 4 we obtain our main result.

Corollary 3. A separable Banach space $E$ has the BAP if and only if $G^{\infty}\left(U_{E}\right)$ has the BAP.

Proof. Let $E$ be a separable Banach space and suppose that $E$ has the BAP. Let $I$ be the identity mapping on $G^{\infty}\left(U_{E}\right)$. Then by Corollary 2 and Proposition 4 there exists a sequence $\left(T_{n}\right)_{n=1}^{\infty} \subset G^{\infty}\left(U_{E}\right)^{\prime} \otimes G^{\infty}\left(U_{E}\right)$ such that $T_{n}(u) \rightarrow I(u)$ for every $u \in G^{\infty}\left(U_{E}\right)$. Since $G^{\infty}\left(U_{E}\right)$ is also a separable Banach space (see [10, Remark 2.2]), by a characterization of the BAP for separable Banach spaces (see, for example, [9, Theorem 27.4]), we conclude that $G^{\infty}\left(U_{E}\right)$ has the BAP. Since the converse implication follows from Proposition 3, the proof is complete.

Hence, the above result provides a positive answer to the problem posed by Mujica in [10] in the case of separable Banach spaces.

\section{References}

[1] E. Çalışkan, Approximation of holomorphic mappings on infinite dimensional spaces, Rev. Mat. Complut. 17 (2004), 411-434.

[2] P. G. Casazza, Approximation properties, in: Handbook of the Geometry of Banach Spaces, Vol. 1, W. Johnson and J. Lindenstrauss (eds.), North-Holland, Amsterdam, 2001, 271-316.

[3] S. Dineen, Complex Analysis on Infinite Dimensional Spaces, Springer Monogr. Math., Springer, Berlin, 1999.

[4] S. Dineen and J. Mujica, The approximation property for spaces of holomorphic functions on infinite dimensional spaces I, J. Approx. Theory 126 (2004), 141-156.

[5] T. Figiel and W. B. Johnson, The approximation property does not imply the bounded approximation property, Proc. Amer. Math. Soc. 41 (1973), 197-200.

[6] J. Lindenstrauss, Weakly compact sets-their topological properties and the Banach spaces they generate, in: Symposium on Infinite Dimensional Topology, R. D. Anderson (ed.), Ann. of Math. Stud. 69, Princeton Univ. Press, 1972, 235-273.

[7] J. Lindenstrauss and L. Tzafriri, Classical Banach Spaces I, Springer, Berlin, 1977.

[8] J. Mujica, Holomorphic approximation in infinite-dimensional Riemann domains, Studia Math. 82 (1985), 107-134.

[9] - Complex Analysis in Banach Spaces, North-Holland Math. Stud. 120, NorthHolland, Amsterdam, 1986.

[10] - Linearization of bounded holomorphic mappings on Banach spaces, Trans. Amer. Math. Soc. 324 (1991), 867-887.

[11] - Reflexive spaces of homogeneous polynomials, Bull. Polish Acad. Sci. Math. 49 (2001), 211-223.

[12] A. Pełczyński, Any separable Banach space with the bounded approximation property is a complemented subspace of a Banach space with a basis, Studia Math. 40 (1971), 239-243. 
[13] R. R. Ryan, Applications of topological tensor products to infinite dimensional holomorphy, Ph.D. thesis, Trinity College, Dublin, 1980.

[14] P. Takatsuka, Normal families of holomorphic functions on infinite dimensional spaces, Portugal. Math. 63 (2006), 351-362.

Yıldız Teknik Üniversitesi

Fen-Edebiyat Fakültesi

Matematik Bölümü

Davutpaşa, 34210 Esenler

Istanbul, Turkey

E-mail: caliskan@yildiz.edu.tr

Received June 30, 2005

Revised version November 3, 2006 